\title{
ON I-DUO RINGS
}

\author{
AMADOU LAMINE FALL AND MAMADOU SANGHARE
}

This paper is dedicated to professor Souleymane NIANG

\section{INTRODUCTION}

Let $R$ be a non commutative associative ring with unit 1 . A left $R$ - module $M$ is said to satisfy property $(I)$ if every injective endomorphism of $M$ is an automorphism. It is well know that every artinian module satisfies property $(I)$, and that the converse is false. A ring $R$ is called left (resp right) I-ring if every left (right) $R$-module with property $(I)$ is artinian. Recall that a ring $R$ is a left (resp right) pure-semi-simple ring if every left (resp right) $R$-module is a direct sum of indecomposables left (right ) $R$-modules . $R$ is of finite representation type if $R$ is left (right) artinian and has finite many non isomorphic indecomposable left (right) $R$-modules . We recall that the concept of finite representation type is left-right symmetric. Following [7] a left $R$-module $M$ is said to have property (S) if every surjective endomorphism of $\mathrm{M}$ is an automorphism; $R$ is called left (right) S-ring if every left(right) $R$-module with property (S) is noetherian . An $R$-module is said to be uniserial if its submodules are linearly ordered by inclusion . $R$ is left (right) serial if it is a direct sum of left (right) uniserial $R$-modules and $R$ is serial if it is both left and right serial. A duo-ring is a ring in which every one sided ideal is two sided. Definitions and notations used in this paper can be found ind [8].

In this paper we prove that for a duo-ring $R$ the following conditions are equivalent.

1. $R$ is a left $I$-ring.

2. $R$ is of finite representation type.

3. $R$ is left pure-semi-simple.

4. $R$ is an artinian principal ideal ring (uniserial).

5. $R$ is a left $S$-ring .

6. $R$ is a right I-ring .

7. $R$ is right pure semi-simple.

8. $R$ is a right S-ring .

\section{Preliminary}

Definition 2.1. Let $R$ be a ring. A left $R$-module ${ }_{R} M$ is said to satisfy property (I) if all of its injective endomorphisms are automorphisms. $R$ is called left (right) $I$-ring if every left (right) $R$-module with property $(I)$ is artinian. $R$ is an I-ring if it is a left and right $I$-ring. We recall that a ring $R$ is a left (right) duo-ring if every left (right) ideal of $R$ is a two sided ideal. A left and right duo-ring is called duo-ring. 
Proposition 2.1. If $R$ is an $I$-duo ring then every prime ideal of $R$ is a maximal ideal and further more the set of all prime ideals is finite.

Proof. Let $P$ be a prime ideal of $R$. the factor ring $R / P$ is an $I$-duo ring which is a domain. Let $K$ be the classical ring of fraction of $R / P ; K$ is a division ring, hence the $R / P$-module $K$ satisfies property $(I)$. It follows that the module $K$ is artinian and that $K=R / P$. Let $\left\{P_{l} / l \in L\right\}$ the set of all prime ideals. If $l \neq m$ then $\operatorname{Hom}\left(R / P_{l}, R / P_{m}\right)=\{0\}$, it follows that the $R$-module $M=\bigoplus_{l \in L} R / P$ satisfies property $(I)$ and hence $L$ is a finite set.

Corollary 2.1. The Jacobson radical $J$ of any $I$-duo ring $R$ is a nil ideal .

Proposition 2.2. Let $R$ be a semi prime duo ring, then every one sided regular element of $R$ is two sided regular.

Proof. Let $x$ a one sided regular element of $R$. Assume that $x$ is a right regular; let $y \in R$ such that $y x=0$ than

$$
(x y)^{2}=x(y x) y=0
$$

since $R$ is semi prime and $x y$ is nilpotent we have $x y=0$ and hence $y=0$.

Proposition 2.3. Let $R$ be a semi prime $I-d u o$ ring; then $R$ is artinian.

Proof. Let $R^{\prime}=S^{-1} R$ be the ring of fraction of $R$ where $S$ is the set of all regular elements of $R$. Any endomorphism of the left $R$-module $R^{\prime}$ is obtained by multiplication by a element of $R^{\prime}$; it follows that the $R$-module $R^{\prime}$ satisfies property $(I)$. Since $R$ is an $I$-ring, then $R \prime$ is an artinian $R$-module and hence $R=R^{\prime}$. So $R$ is artinian.

Theorem 2.1. Let $R$ be an $I-d u o$ ring; then $R$ is artinian.

Proof. It follows from corollary 2.1 that the Jacobson radical $J$ of $R$ is a nil ideal. Then every idempotent of $R / J$ can be lifted to a idempotent of $R$. Since $R / J$ is a semi prime $I$-duo ring, it result from proposition 2.3 is semi simple; and that $R$ is a semi-perfect ring. So $R$ can be written

$$
R=\operatorname{Re}_{1} \bigoplus \operatorname{Re}_{2} \bigoplus \ldots . . \bigoplus R e_{n},
$$

where the $e_{i}{ }^{\prime} s$ are central idempotents and each $R e_{i}$ is a local projectif R-module. To prove that $R$ is artinian, it is sufficient to prove that each $R e_{i}$ is artinian as left $R$-module. Let $f \neq 0$ be an non surjective endomorphism of $R e_{i}$. We have $f\left(R e_{i}\right) \subseteq J e_{i}$, let us put $f\left(e_{i}\right)=r e_{i} ; r \in J$. As $J$ is a nil ideal, there exists an integer $n \in N^{*}$ such that $r^{n} e_{i} \neq 0$ and $r^{n+1} e_{i}=0$. For this integer we have $f\left(r^{n} e_{i}\right)=r^{n} f\left(e_{i}\right)=r^{n+1} e_{i}=0$. It follows that $\mathrm{f}$ is not monic. So the $R$-module $R e_{i}$ satisfies property $(I)$. Hence $R e_{i}$ is artinian.

Remark 2.1. (a) Every artinian duo-ring is a finite direct product of artinian local duo-rings (b) It is proved in [3] that if $R$ is an artinian local duo ring with

Jacobson radical $J$, then $R$ is uniserial or $R / J$ is a field. 


\section{Characterization of I-Duo Rings}

In what follows $R$ will denote an artinian local duo-ring with Jacobson radical $J$ satisfying $J^{2}=0$. It results then from remarks 2.1 that if $R$ has a non principal ideal, then $R / J$ is a field. We have then two cases : Case $1: R / J$ is an infinite field and $\operatorname{dim}_{R / J} J / J^{2}=2$ Let $H$ be a complete set of representants of $(R / J) \backslash\{\overline{0}\} H$ is a infinite set. For $h \in H$, set $I=R\left(x_{1}-h x_{2}\right)$ where $\left\{\bar{x}_{1}, \bar{x}_{2}\right\}$ is a basis of $J / J^{2}$ over $R / J$; and $M_{h}=R / I_{h}$.

Lemma 3.1. If $h \neq h^{\prime}$ are in $H$ then $x_{1}-h x_{2} \notin I_{h^{\prime}}$

Proof. Assume that $x_{1}-h x_{2} \in I_{h^{\prime}}=R\left(x_{1}-h x_{2}\right)$. Let $\alpha \in R \backslash J$ such that $x_{1}-h x_{2}=\alpha\left(x_{1}-h^{\prime} x_{2}\right)$ then $(1-\alpha) x_{1}-\left(h-\alpha h^{\prime}\right)=0$, it follows that $1-\alpha \in J$ and $h-\alpha h^{\prime} \in J$. Let $m \in J$ such that $\alpha=1+m$. Since $h-(1+m) h^{\prime} \in J$, we have $h-h^{\prime} \in J$ which contradicts the choice of $H$.

Lemma 3.2. Let $h, h^{\prime} \in H, h \neq h^{\prime}$. If $g: M_{h} \longrightarrow M_{h^{\prime}}$ is an homomorphism of $R$-modules then $g\left(1+I_{h}\right)$ is note invertible in the ring $M_{h^{\prime}}$. So $g\left(1+I_{h}\right) \in J / I_{h^{\prime}}$

Notation 3.1. If $x \in R$ and $h \in H$, we set $x+I_{h}=x_{M_{h}}$.

Proof of lemma 3.2. We have

$$
0_{M_{h^{\prime}}}=g\left(0_{M_{h}}\right)=g\left[\left(x_{1}-h x_{2}\right)+I_{h}\right]=\left(x_{1}-h x_{2}\right) g\left(1+I_{h}\right),
$$

hence $g\left(1+I_{h}\right)$ is not invertible in $M_{h^{\prime}}$ so $g\left(1+I_{h}\right) \in J / I_{h^{\prime}}$.

Corollary 3.1. Let $f: \bigoplus_{h \in H} M_{h} \longrightarrow \underset{h \in H}{\bigoplus} M_{h}$ be an endomorphism of the $R$-module $\bigoplus_{h \in H} M_{h}$. If $i_{h}$ and $p_{h^{\prime}}$ are respectively the canonical injection of $M_{h}$ in $\bigoplus_{k \in H} M_{k}$ and the canonical projection of $\bigoplus_{h \in H} M_{h}$ on $M_{h^{\prime}}$, then $p_{h} \circ f \circ i_{h}\left(1+I_{h}\right) \in J / I_{h^{\prime}}$.

If $x \in \bigoplus_{h \in H} M_{h}=M$, we note $x=\sum_{h \in H} \alpha_{h} e_{h}$ where $e_{h}=1+I_{h}$ and $\alpha_{h} \in R$, and $f\left(e_{h}\right)=\sum_{h^{\prime} \in H}^{h \in H} \beta_{h^{\prime}} e_{h^{\prime}}$ where $\beta_{h^{\prime}} e_{h^{\prime}}=p_{h^{\prime}}^{h \in H} f \circ i_{h}\left(e_{h}\right)$. So $\beta_{h^{\prime}} \in J$ if $h \neq h^{\prime}$. Let $\mathrm{f}$ be an injective endomorphism of $M=\bigoplus_{h \in H} M_{h}$ we have the following lemmas.

Lemma 3.3. For every $h \in H, f\left(e_{h}\right)=\beta_{h} e_{h}+\sum_{h \neq h^{\prime}} \beta_{h^{\prime}} e_{h^{\prime}}$; where $\beta_{h} \notin J$.

Proof. Let $h \in H$. If $h^{\prime} \in H$ and $h^{\prime} \neq h$ then, by lemma 3.1, we have

$$
0_{M} \neq f\left[\left(x_{1}-h^{\prime} x_{2}\right) e_{h}\right]=\left(x_{1}-h^{\prime} x_{2}\right) \beta_{h} e_{h},
$$

it follows that $\beta_{h} \notin J$.

Lemma 3.4. J.M $\subseteq \operatorname{Imf}$.

Proof. Let $m$ be an element of $J$. For $h \in H$, we have $f\left(m e_{h}\right)=m \beta_{h} e_{h}$. Since $R$ is a duo ring and $\beta_{h} \notin J$, there exists $\beta_{h}^{\prime} \in R \backslash J$ such that $m \beta_{h}=\beta_{h}^{\prime} m$; we have then $m e_{h}=f\left(\beta_{h}^{\prime-1} m e_{h}\right)$. So $m e_{h} \in \operatorname{Imf}$ and hence $J . M \subseteq \operatorname{Imf}$.

Lemma 3.5. For every $h \in H \quad, e_{h} \in \operatorname{Imf}$. 
Proof. Let $h \in H$. By lemma 3.3 we have

$$
f\left(e_{h}\right)=\beta_{h} e_{h}+\sum_{h \neq h^{\prime}} \beta_{h^{\prime}} e_{h^{\prime}} \beta_{h} \notin J \text { and } \beta_{h^{\prime}} \in J, \text { for } h \neq h^{\prime} .
$$

Then $\beta_{h} e_{h}=f\left(e_{h}\right)-\sum_{h \neq h} \beta_{h^{\prime}} e_{h^{\prime}}$, so $e_{h}=f\left(\beta_{h}^{-1} e_{h}\right)-\sum_{h \neq h^{\prime}} \beta_{h}^{-1} \beta_{h}^{\prime} e_{h^{\prime}}$. Since $f\left(\beta_{h}^{-1} e_{h}\right)$ and $\sum_{h \neq h^{\prime}} \beta_{h}^{-1} \beta_{h}^{\prime} e_{h^{\prime}}$ are in $\operatorname{Imf}$ then $e_{h} \in \operatorname{Imf}$.

We can now state the following assertion.

Theorem 3.1. Let $R$ be a local artinian duo ring with maximal ideal J such that $J^{2}=$ 0 . If $R / J$ is an infinite field and $\operatorname{dim}_{R / J} J / J^{2} \geq 2$ then there exists an non artinian $R$-module $M$ with property $(I)$.

Case2: We assume that $R / J$ is a finite field and that $\operatorname{dim}_{R / J} J / J^{2}=2$. In this

case the characteristic of the field $R / J$ is a prime number $p$ and the characteristic of $R$ is $p$ or $p^{2}$. and hence $R / J$ is a separable finite extension of $Z(R) / J \cap Z(R)$ where $Z(R)$ is the center of $R$. It follows from [6] that there exists an artinian principal ideal subring $B$ of $R$ such that $R=B \bigoplus B c$ as $B$ - modules, where $c \in J$. So let us set $B b$ the Jacobson radical of $B$, we have $b^{2}=b c=c^{2}=0$. In what follows homomorphism will be in the opposite side of the scalars. Let

$$
\begin{array}{r}
M_{R}=R_{R}^{\left(N^{*}\right)}=\bigoplus_{i \in N^{*}} e_{i} R \text { where } e_{i}=\left(\delta_{i}^{j}\right)_{j \in N^{*}} \text { and } \\
\delta_{i}^{j}=\left\{\begin{array}{c}
1_{R}, \text { if } i=j \\
i_{0_{R}}, \text { if } i \neq j
\end{array}\right.
\end{array}
$$

and let $\sigma: M_{R} \longrightarrow M_{R}$ be the endomorphism of $M_{R}$ given by :

$$
\sigma\left(e_{i}\right)=\left\{\begin{array}{c}
0, \text { if } i=1 \\
e_{i-1}, \text { if } i \geq 2
\end{array}\right.
$$

If $z \in R$ we denote $L_{z}$ the endomorphism of $M_{R}$ defined for $m \in M_{R}$ by $L_{z}(m)=z m$. Let $\Lambda$ be the subring of $E n d M_{R}$ generated by $d=L_{c} \circ \sigma$ and the elements $L_{x}, x \in B$. By the ring homomorphism

$$
\begin{gathered}
R=B \oplus B b \longrightarrow \Lambda \\
x+y b \longrightarrow L_{x}+L_{y} \circ d .
\end{gathered}
$$

M has a structure of left $R$ - module defined as follows

$$
(x+y c) m=\left(L_{x}+L_{y} \circ d\right)(m) .
$$

Let now $f$ be an injective endomorphism of ${ }_{R} M$, we have $(d . m) f=d .(m) f$ for $m \in M$. We shall prove the following lemmas.

Lemma 3.6. For every $n \in \mathbb{N}^{*}$, we have $d\left(e_{n}\right) f=\left(c e_{n-1}\right) f$, for $n \geq 2$, and $0=d\left(e_{1}\right) f$. 
Lemma 3.7. For every $n \in \mathbb{N}^{*}$, we have

$$
\left(e_{n}\right) f=\sum_{i n} \alpha_{k, n} e_{k}
$$

where $\alpha_{n, n}$ is invertible in $R$, and $\alpha_{k, n} \in J$ for $k>n$.

Proof. Set $\left(e_{1}\right) f=\alpha_{1,1} e_{1}+\sum_{i>1} \alpha_{i, 1} e_{i}$. Since $c e_{1} \neq 0$, then

$$
c\left(\alpha_{1,1}+\sum_{i>1} \alpha_{i, 1} e_{i}\right)=\left(c e_{1}\right) f \neq 0
$$

But $c\left(\sum_{i>1} \alpha_{i, 1} e_{i-1}\right)=c \sigma\left(\alpha_{1,1} e_{1}+\sum_{1<1} \alpha_{i, 1} e_{i}\right)=c \sigma\left[\left(e_{1}\right) f\right]=\left(c \sigma e_{1}\right) f=(0) f=0(2)$.

So by (2), $\alpha_{i, 1} \in J$ fori $>1$ and by $(1) \alpha_{1,1} \notin J$.

Suppose now that

$$
\left(e_{n-1}\right) f=\sum_{i<n-1} \alpha_{i, n-1} e_{i}+\alpha_{n-1, n-1} e_{n-1}+\sum_{i>n-1} \alpha_{i, n-1} e_{i}
$$

where $\alpha_{n-1, n-1} \notin J$. and $\alpha_{i, n-1} \in J$ for $i>n-1$; and let us set $\left(e_{n}\right) f=$ $\sum_{i \geq 1} \alpha_{i, n} e_{i}$. Then

$$
c \sigma\left(\sum_{i \geq 1} \alpha_{i, n} e_{i} .\right)=c \sigma\left(e_{n}\right) f=c\left(e_{n-1}\right) f=\left(c e_{n-1}\right) f \neq 0 .
$$

Since $c\left(\sum_{i \geq 2} \alpha_{i, n} e_{i-1}.\right)=c \sigma\left(e_{n}\right) f=c\left(e_{n-1}\right) f=\sum_{i<n-1} c \alpha_{i, n-1} e_{i}+c \alpha_{n-1, n-1} e_{n-1}$, where $c \alpha_{n-1, n-1} e_{n-1} \neq 0$, then $c \alpha_{n, n} \neq 0$ and $c \alpha_{i, n}=0$ for $i>n$. It follows then that $\alpha_{n, n} \notin J$ and $\alpha_{i, n} \in J$ for $i>n$.

Lemma 3.8. For every $n \in \mathbb{N}^{\star}$, we have J.e $e_{n} \subseteq \operatorname{Imf}$.

Proof. Let $m \in J$ we have $\left(m e_{1}\right) f=m\left(e_{1}\right) f=m \alpha_{1,1} e_{1}$. Let $\quad \alpha_{1,1}^{\prime} \in R \backslash J$ such that $m \alpha_{1,1}=a_{1,1}^{\prime} m$, then $\left(m e_{1}\right) f=\alpha_{1,1}^{\prime} m e_{1}$ hence $m e_{1}=\left(\alpha_{1,1}^{\prime}\right)^{-1}\left(m e_{1}\right) f \in I m f$.

Suppose that $J e_{k} \subseteq \operatorname{Imf}$ for $k \leq n-1$ and let $m \in J$, we have :

$$
\left(m e_{n}\right) f=\sum_{i, 1} c_{i, 1} e_{i}
$$

where $\alpha_{1,1} \notin J$ and $\alpha_{i, 1} \in J$ for $i>1$.

Assume that for every $k<n, e_{k} \in \operatorname{Im} f$. Since

$$
\left(e_{n}\right) f=\sum_{\in} c_{k, n} e_{k}
$$

where $\alpha_{n, n}$ is invertible and $c_{k, n} \in J$, we have

$$
\alpha_{n, n} e_{n}=\left(e_{n}\right) f-\sum_{\in} c_{k, n} e_{k} \in \operatorname{Im} f
$$

and so

$$
e_{n}\left(\alpha_{n, n}^{-1} e_{n}\right) f-\sum_{n>i} \alpha_{i, n}^{-1} e_{i}-\bigoplus_{n n} J e_{i}
$$

of ${ }_{R} M$ is strictly decreasing.

We have proved the following result : 
Theorem 3.2. Let $R$ be an artinian local duo ring with maximal ideal $J$ such that $J^{2}=(0)$. If $R / J$ is a finite field and $\operatorname{dim}_{R / J} J / J^{2} \geq 2$ then there exists a non artinian $R$-module with property $(I)$.

We have the following theorem :

Theorem 3.3. Let $R$ be a duo ring. The following statements are equivalent.

1. $R$ is a left $I$-ring.

2. $R$ is an uniserial ring.

3. $R$ is a left $S$-ring.

4. $R$ is a left pure semi-simple ring.

5. $R$ has a finite representation type.

6. $R$ is a right $I$-ring.

7. $R$ is a right $S-$ ring.

8. $R$ is a right pure semi-simple ring.

Proof. It suffices to proved the equivalence 1)

$1) \Longrightarrow 2$ ). By theorem $2.1 \quad R$ is Artinian and by theorem 3.1 and theorem 3.2 $R$ is necessarily a principal ideal ring .

$(2) \Longrightarrow(1)$ If $R$ is an uniserial ring than every left $R$-module is a direct sum of cyclic modules . Let $M$ be an artinian $R$ - module, since there is only finite non isomorphic cyclic $R$-modules we can write $M=K^{\left(N^{*}\right)} \bigoplus L$ where $K$ is cyclic submodule of $M$. Since $K^{\left(N^{*}\right)}$ does not satisfy property $(I)$, it follows that $M$ does not satisfy property $(I)$.

\section{REFERENCES}

1. Anderson, F.W. and Fuller, K.R., Rings and categories of modules, Springer Verlag (1973).

2. Courter, R.C., finite dimensional right duo algebras are duo, Prooc . A.M.S 84(02) 1982, 157-161.

3. Habeb, J., on azumaya 's exact rings and artinian duo rings .comm., in algebra 17(1) (1989) 237-245.

4. Kaidi, A.M. et Sanghare, M., une caracterisation des anneaux artiniens à idéaux pricipaux, Lec.Notes in Maths 1328, Springer -verlag, 245-254

5. Leradji, A. On duo rings, pure-semi simplicity and finite representation type, comm.in algebra 25(12) (1997) 3947-3952.

6. Pop, H.C., On the structure of artinian rings, comm.in algebra 15(11) (1987), 2327-2348.

7. Sanghare, M., On S-duo rings, comm. in algebra 20(8) (1992) 2183-2189.

8. WEIMIN XUE, Rings with Morita duality, Lec. Notes in Maths, 1523, Springer-Verlag(1992).

Département de Mathématique et informatique, Faculté des Sciences et Techniques, Université Cheikh Anta Diop de Dakar, Sénégal 